\title{
6-Methyl-2,4-pyrimidyl Diesters. A Highly Efficient Acylating Agent for the Synthesis of Unsymmetrical Ketones ${ }^{\dagger}$
}

\author{
Jae In Lee \\ Department of Chemistry and Plant Resources Research Institute, College of Natural Science, Duksung Women's University, \\ Seoul 132-714, Korea.E-mail: jilee@duksung.ac.kr \\ Received January 9, 2010, Accepted February 1, 2010
}

Key Words: 6-Methyl-2,4-pyrimidyl diesters, Acylating agents, Ketones, Substitution

It is well known that the reaction of organometallics with carboxylic acid derivatives is a useful method for the synthesis of ketones. ${ }^{1}$ The reaction of acid chlorides with Grignard reagents gives ketones, but yields are low to moderate due to the formation of the corresponding tertiary alcohols. ${ }^{2}$ To overcome these drawbacks the reaction of in situ formed acyl triphenylphosphonium ions from acid chlorides and $\mathrm{Bu}_{3} \mathrm{P}$ with Grignard reagents has been developed, but the separation of $\mathrm{Bu}_{3} \mathrm{P}$ is tedious. ${ }^{3}$ Alternatively, the coupling of acid chlorides with terminal alkynes $^{4}$ or organostannanes ${ }^{5}$ in the presence of palladium catalyst and alkynyldimethylaluminum reagents, ${ }^{6}$ derived from $\mathrm{Et}_{3} \mathrm{~N}$-catalyzed alumination of terminal alkynes, leads to the aromatic ketones or ynones at reflux temperature. Although thiol esters are also coupled with organozinc ${ }^{7}$ or organoboron reagents ${ }^{8}$ in the presence of $\mathrm{PdCl}_{2}\left(\mathrm{PPh}_{3}\right)_{2}$ to afford ketones including aldehyde, ester, and halide functional groups, these methods are exclusively limited to the synthesis of aliphatic ketones.

The reaction of tertiary amides such as $N$-acylaziridines ${ }^{9}$ and morpholine amides ${ }^{10}$ with Grignard or organolithium reagents gives ketones upon acidic workup of the tetrahedral intermediates in moderate to high yields. Among tertiary amides, $N$-methoxy- $N$-methylamides ${ }^{11}$ developed by Weinreb is most popular, which are converted to ketones with both organolithiums and Grignard reagents through metal-chelated intermediates. ${ }^{12}$ However, the high cost of $\mathrm{MeONHMe} \cdot \mathrm{HCl}$ and low yields in case of less reactive Grignard reagents make Weinreb amides to limit their uses. The most effective acylating agents toward Grignard reagents appear to be the active esters containing 2-pyridyl moiety which enhances the reactivity and prevents the release of carbonyl group by ring nitrogen atom. Thus, the treatment of $S$-(2-pyridyl) thioates ${ }^{13}$ and $N$-methyl- $N$-(2-pyridyl) amides ${ }^{14}$ with Grignard reagents leads to the 6-membered chelates, which prevent the premature ketones liberation, and are dissociated to afford ketones. The reaction of 2-acyl-3-methylpyrazines ${ }^{15}$ and $N$-(acyl- $N$-methylamino)cycloiminium salts ${ }^{16}$ with Grignard or organolithium reagents also gives ketones, but 2 equiv of organometallics is required for the high yields formation of ketones.

However, there are no reports of acylating agents that can produce 2 equiv of ketones with ogranometallics except our previous report. ${ }^{17}$ In conjunction with the development of new acylating agents we wish to report that 2 equiv of ketones can

${ }^{\dagger}$ This paper is dedicated to Professor Sunggak Kim on the occasion of his honorable retirement. be efficiently synthesized from 6-methyl-2,4-pyrimidyl diesters and Grignard reagents in high yields. In choosing 6-methyl2,4-pyrimidyl group as active moiety, we considered that two nitrogen atoms of ring are chelated with magnesium atoms, hence to avoid the formation of the corresponding tertiary alcohol. Furthermore, 6-methyl-2,4-pyrimidyl diesters are the stable crystalline compounds, reactive toward Grignard reagents, and 2,4-dihydroxy-6-methylpyrimidine as a starting material is very cheap.

6-Methyl-2,4-pyrimidyl diesters 3 were newly prepared by the addition of 2 equiv of acid chlorides 2 to a mixture solution of 2,4-dihydroxy-6-methylpyrimidine 1 and 2 equiv of triethylamine in dichloromethane at room temperature (Scheme 1). The nucleophilic acyl substitution of $\mathbf{2}$ with $\mathbf{1}$ was completed in $3 \sim 12 \mathrm{~h}$ at room temperature because 1 was slightly soluble in dichloromethane. After evaporation of dichloromethane, the mixture was dissolved in THF, followed by filtering off triethylamine hydrochloride and was separated by usual workup. The active esters 3 were obtained in $88 \sim 95 \%$ yields after recrystallization from $15 \sim 20 \% \mathrm{EtOAc} / n$-hexane and are thermally stable, thus their melting points were measured without any decomposition.

The successful synthesis of ketones $\mathbf{5}$ using $\mathbf{3}$ was carried out by the slow addition of 2 equiv of Grignard reagents to a solution of $\mathbf{3}$ in THF at $-15{ }^{\circ} \mathrm{C}$. For example, 2 equiv of $2,4,6-$ trimethylphenylmagnesium bromide was added to a solution of 1 equiv of 6-methyl-2,4-pyrimidyl dibenzoate in THF over $5 \mathrm{~min}$ at $-15^{\circ} \mathrm{C}$ and stirred for $3 \mathrm{~h}$ between $-15^{\circ} \mathrm{C}$ and room

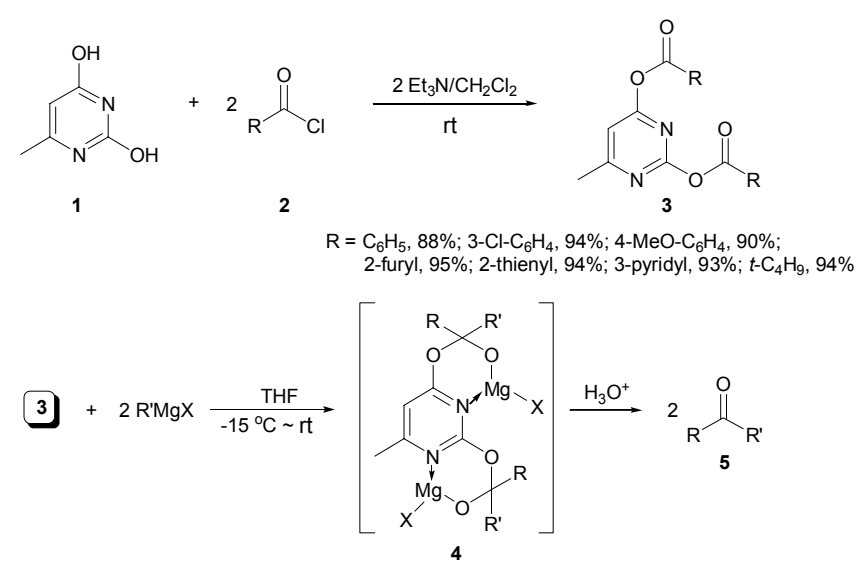

Scheme 1 
Table 1. Preparation of unsymmetrical ketones from 6-methyl-2,4-pyrimidyl diesters and Grignard reagents

\begin{tabular}{|c|c|c|c|c|c|}
\hline \multirow{2}{*}{ entry 5} & \multirow{2}{*}{$\mathrm{R}$} & \multirow{2}{*}{$\mathrm{R}^{\prime} \mathrm{MgX}$} & reaction conditions & \multirow{2}{*}{ ketones } & \multirow{2}{*}{$\begin{array}{c}\text { isolated yield } \\
(\%)\end{array}$} \\
\hline & & & temp $\left({ }^{\circ} \mathrm{C}\right)$; time $(\mathrm{h})$ & & \\
\hline a & $\mathrm{C}_{6} \mathrm{H}_{5}$ & & $-15 ; 0.5$ & & 85 \\
\hline b & & $\mathrm{MgBr}$ & $-15 \rightarrow \mathrm{rt} ; 2$ & & 73 \\
\hline c & & & $-15 \rightarrow \mathrm{rt} ; 3$ & & 95 \\
\hline d & $3-\mathrm{Cl}-\mathrm{C}_{6} \mathrm{H}_{4}$ & & $\mathrm{rt} ; 7$ & & 84 \\
\hline e & & & $-15 \rightarrow 0 ; 3$ & & 91 \\
\hline f & $4-\mathrm{MeO}-\mathrm{C}_{6} \mathrm{H}_{4}$ & $\mathrm{CH}_{3} \mathrm{MgBr}$ & $-15 \rightarrow 0 ; 1$ & & 78 \\
\hline g & & & $-15 \rightarrow 0 ; 1$ & & 94 \\
\hline $\mathbf{h}$ & 2-Furyl & & $-15 \rightarrow \mathrm{rt} ; 1$ & & 87 \\
\hline $\mathbf{i}$ & 2-Thienyl & & $-15 \rightarrow \mathrm{rt} ; 0.5$ & & 81 \\
\hline $\mathbf{j}$ & & & $-15 \rightarrow \mathrm{rt} ; 1.5$ & & 82 \\
\hline $\mathbf{k}$ & 3-Pyridyl & & $-15 \rightarrow \mathrm{rt} ; 1$ & & 90 \\
\hline 1 & & & $-15 \rightarrow \mathrm{rt} ; 1$ & & 87 \\
\hline $\mathbf{m}$ & $\left(\mathrm{CH}_{3}\right)_{3} \mathrm{C}$ & & $-15 \rightarrow 0 ; 1$ & & 81 \\
\hline $\mathbf{n}$ & & & $0 \rightarrow \mathrm{rt} ; 6$ & & 78 \\
\hline
\end{tabular}

temperature. The mixture was hydrolyzed with saturated $\mathrm{NH}_{4} \mathrm{Cl}$ solution to give 2,4,6-trimethylbenzophenone in 95\% yield without the formation of the corresponding tertiary alcohol. The success of ketone synthesis may be ascribed to the formation of 6-membered chelate 4 between magnesium atoms of Grignard reagent and carbonyl oxygen/ring nitrogen atoms of 3, which would react very sluggishly with Grignard reagent and is dissociated to give $\mathbf{5}$ after acidic hydrolysis.
As shown in Table 1, various unsymmetrical ketones were efficiently prepared in high yields $(73 \sim 95 \%)$ by this method. The reaction worked well with both aliphatic and aromatic Grignard reagents regardless of the kind of electron donating $(\mathbf{5 e}, \mathbf{5 j}, \mathbf{5 l})$ and electron withdrawing group $(\mathbf{5 g}, \mathbf{5 h})$ in the substituted phenylmagnesium bromide. Also the kind of electron withdrawing (5d, 5e) and electron donating group (5f, 5g) in the substituted 6-methyl-2,4-pyrimidyl dibenzoate didn't in- 
fluence on the substitution of 6-methyl-2,4-pyrimidyl group by Grignard reagents. The reaction proceeded equally well with 6-methyl-2,4-pyrimidyl diesters containing heteroaromatic groups (5h-5l) to give the corresponding ketones in high yields $(81 \sim 90 \%)$ within $1.5 \mathrm{~h}$ between $-15^{\circ} \mathrm{C}$ and room temperature. Although the reaction of $\mathbf{3}$ with 1-hexynylmagnesium bromide (5b) and highly hindered 2,4,6-trimethylphenylmagnesium bromide $(\mathbf{5 c}, \mathbf{5 n})$ proceeded slowly between $-15^{\circ} \mathrm{C}$ and room temperature, the corresponding ketones were obtained in $73 \%$, $95 \%$, and $78 \%$ yield, respectively. Especially, it was worth while to note that the reaction of 6-methyl-2,4-pyrimidyl di(3-chlorobenzoate) with (1,3-dioxolan-2-ylmethyl)magnesium bromide afforded $\alpha$-(1,3-dioxolan-2-yl) 3'-chloroacetophenone 5d in $84 \%$ yield in $7 \mathrm{~h}$ at room temperature. The corresponding reaction using $N$-methoxy- $N$-methyl 3-chlorobenzamide didn't proceed to give $\mathbf{5 d}$ even after $24 \mathrm{~h}$ at room temperature and the starting material was recovered in $98 \%$ yield.

In summary, 6-methyl-2,4-pyrimidyl diesters are newly developed by the reaction of 2 equiv of acid chlorides and cheap 2,4-dihydroxy-6-methylpyrimidine, are stable for long periods of time, and react efficiently with Grignard reagents to give 2 equiv of ketones in high yields under mild conditions, and thus may be utilized in many ketone syntheses.

\section{Experimental Section}

Preparation of 6-methyl-2,4-pyrimidyl dibenzoate (General procedure). To a suspension of 2,4-dihydroxy-6-methylpyrimidine $(883 \mathrm{mg}, 7.0 \mathrm{mmol})$ in dichloromethane $(50 \mathrm{~mL})$ was added triethylamine $(1.97 \mathrm{~mL}, 14.1 \mathrm{mmol})$ and benzoyl chloride $(1.97 \mathrm{~g}, 14.0 \mathrm{mmol})$ at room temperature. After being stirred overnight, dichloromethane was evaporated in vacuo. The mixture was dissolved in THF, followed by filtering off triethylamine hydrochloride. After evaporation of THF, the mixture was poured into saturated $\mathrm{NaHCO}_{3}$ solution $(40 \mathrm{~mL})$, extracted with dichloromethane $(3 \times 30 \mathrm{~mL})$, and washed with brine $(40$ $\mathrm{mL})$. The combined organic phases were dried over $\mathrm{MgSO}_{4}$, filtered, and concentrated in vacuo. The residue was recrystallized from $15 \% \mathrm{EtOAc} / n$-hexane to give 6-methyl-2,4-pyrimidyl dibenzoate $(2.06 \mathrm{~g}, 88 \%)$. mp $82 \sim 83{ }^{\circ} \mathrm{C} ;{ }^{1} \mathrm{H}$ NMR $(300$ $\left.\mathrm{MHz}, \mathrm{CDCl}_{3}\right) \delta 8.19-8.24(\mathrm{~m}, 4 \mathrm{H}), 7.62-7.70(\mathrm{~m}, 2 \mathrm{H}), 7.48-$ $7.54(\mathrm{~m}, 4 \mathrm{H}), 7.25(\mathrm{~s}, 1 \mathrm{H}), 2.66(\mathrm{~s}, 3 \mathrm{H}) ;{ }^{13} \mathrm{C} \mathrm{NMR}(300 \mathrm{MHz}$, $\left.\mathrm{CDCl}_{3}\right) \delta 173.6,167.0,164.0,163.1,161.0,134.6,134.2,130.6$ (overlapped), 128.8, 128.6, 128.4, 128.0, 110.6, 24.3; FT-IR (KBr) 3068, $1748(\mathrm{C}=\mathrm{O}), 1595,1450,1259,997,699 \mathrm{~cm}^{-1}$; Ms $m / z(\%) 334\left(\mathrm{M}^{+}, 1\right), 306$ (22), 278 (16), 229 (21), 105 $\left(\mathrm{C}_{6} \mathrm{H}_{5} \mathrm{C} \equiv \mathrm{O}^{+}, 100\right), 77$ (92).

Preparation of 1-phenyl-2-heptyn-1-one 5b (General procedure). To a solution of 1-hexyne $(345 \mu \mathrm{L}, 3.0 \mathrm{mmol})$ in THF $(5 \mathrm{~mL})$ was slowly added methylmagnesium bromide $(0.5 \mathrm{M}$ in THF, $6.0 \mathrm{~mL}, 3.0 \mathrm{mmol}$ ) at $0{ }^{\circ} \mathrm{C}$. After being stirred for $0.5 \mathrm{~h}$, the resulting solution was added to a solution of 6-methyl-2,4pyrimidyl dibenzoate $(501 \mathrm{mg}, 1.5 \mathrm{mmol})$ in THF $(15 \mathrm{~mL})$ over $5 \mathrm{~min}$ at $-15^{\circ} \mathrm{C}$. After being stirred for $2 \mathrm{~h}$ between $-15^{\circ} \mathrm{C}$ and room temperature, the mixture was quenched with saturated $\mathrm{NH}_{4} \mathrm{Cl}$ solution $(3 \mathrm{~mL}$ ) and THF was evaporated in vacuo. The mixture was poured into saturated $\mathrm{NH}_{4} \mathrm{Cl}$ solution $(30 \mathrm{~mL})$, extracted with dichloromethane $(3 \times 20 \mathrm{~mL})$, and washed with saturated $\mathrm{NaHCO}_{3}$ solution $(30 \mathrm{~mL})$. The combined organic phases were dried over $\mathrm{MgSO}_{4}$, filtered, and concentrated in vacuo. The residue was purified by vacuum distillation using Kugelrohr apparatus to give $\mathbf{5 b}(408 \mathrm{mg}, 73 \%)$. bp $95 \sim 100{ }^{\circ} \mathrm{C} /$ $1.0 \mathrm{mmHg} ;{ }^{1} \mathrm{H} \mathrm{NMR}\left(300 \mathrm{MHz}, \mathrm{CDCl}_{3}\right) \delta 8.14(\mathrm{~d}, J=8.4 \mathrm{~Hz}$, $2 \mathrm{H}), 7.57-7.62(\mathrm{~m}, 1 \mathrm{H}), 7.45-7.50(\mathrm{~m}, 2 \mathrm{H}), 2.51(\mathrm{t}, J=7.0 \mathrm{~Hz}$, $2 \mathrm{H}), 1.62-1.69(\mathrm{~m}, 2 \mathrm{H}), 1.47-1.55(\mathrm{~m}, 2 \mathrm{H}), 0.97(\mathrm{t}, J=7.3 \mathrm{~Hz}$, $3 \mathrm{H}) ;{ }^{13} \mathrm{C} \mathrm{NMR}\left(300 \mathrm{MHz}, \mathrm{CDCl}_{3}\right) \delta 178.3,136.9,133.9,129.6$, 128.5, 96.9, 79.7, 29.9, 22.1, 18.9, 13.5; FT-IR (film) 3060, 2959, $2200(\mathrm{C} \equiv \mathrm{C}), 1644(\mathrm{C}=\mathrm{O}), 1597,1449,1265,701 \mathrm{~cm}^{-1}$; Ms $m / z(\%) 186\left(\mathrm{M}^{+}, 10\right), 185(23), 157(44), 144\left(\left[\mathrm{C}_{6} \mathrm{H}_{5}-\mathrm{CO}-\mathrm{C} \equiv \mathrm{C}-\right.\right.$ $\left.\left.\mathrm{CH}_{3}\right]^{+}, 100\right), 115$ (55), 105 (74), 77 (46).

3-Methylbutyrophenone (5a): ${ }^{1} \mathrm{H} \mathrm{NMR}\left(300 \mathrm{MHz}, \mathrm{CDCl}_{3}\right)$ $\delta 7.95(\mathrm{~d}, J=8.3 \mathrm{~Hz}, 2 \mathrm{H}), 7.52-7.55(\mathrm{~m}, 1 \mathrm{H}), 7.43-7.48(\mathrm{~m}$, $2 \mathrm{H}), 2.83(\mathrm{~d}, J=6.9 \mathrm{~Hz}, 2 \mathrm{H}), 2.25-2.34(\mathrm{~m}, 1 \mathrm{H}), 1.00(\mathrm{~d}, J=6.7$ $\mathrm{Hz}, 6 \mathrm{H}) ;{ }^{13} \mathrm{C}$ NMR $\left(300 \mathrm{MHz}, \mathrm{CDCl}_{3}\right) \delta 200.3,137.4,132.9$, 128.5, 128.1, 47.5, 25.2, 22.8; FT-IR (film) 3062, 2958, 2871, $1686(\mathrm{C}=\mathrm{O}), 1598,1449,1366,1213,752,691 \mathrm{~cm}^{-1}$; Ms $\mathrm{m} / \mathrm{z}$ (\%) $162\left(\mathrm{M}^{+}, 29\right), 120$ (40), 107 (37), 105 (100), 77 (53).

2,4,6-Trimethylbenzophenone (5c): ${ }^{1} \mathrm{H}$ NMR $(300 \mathrm{MHz}$, $\left.\mathrm{CDCl}_{3}\right) \delta 7.80(\mathrm{~d}, J=8.5 \mathrm{~Hz}, 2 \mathrm{H}), 7.54-7.59(\mathrm{~m}, 1 \mathrm{H}), 7.40-$ $7.46(\mathrm{~m}, 2 \mathrm{H}), 6.89(\mathrm{~s}, 2 \mathrm{H}), 2.33(\mathrm{~s}, 3 \mathrm{H}), 2.08(\mathrm{~s}, 6 \mathrm{H}) ;{ }^{13} \mathrm{C} \mathrm{NMR}$ $\left(300 \mathrm{MHz}, \mathrm{CDCl}_{3}\right) \delta 200.8,138.5,137.3,136.9,134.2,133.6$, 129.4, 128.8, 128.3, 21.2, 19.4; FT-IR (film) 3061, 2920, 1671 $(\mathrm{C}=\mathrm{O}), 1611,1596,1448,1379,1268,1170,910,711 \mathrm{~cm}^{-1}$; Ms $m / z(\%) 224\left(\mathrm{M}^{+}, 83\right), 223$ (100), 209 (43), 147 (51), 119 (27), 105 (16), 77 (28).

$\boldsymbol{\alpha}$-(1,3-Dioxolan-2-yl) 3'-chloroacetophenone (5d): ${ }^{1} \mathrm{H}$ NMR $\left(300 \mathrm{MHz}, \mathrm{CDCl}_{3}\right) \delta 8.03(\mathrm{t}, J=1.7 \mathrm{~Hz}, 1 \mathrm{H}), 7.94\left(\mathrm{dt}, J_{1}=7.8 \mathrm{~Hz}\right.$, $\left.J_{2}=1.3 \mathrm{~Hz}, 1 \mathrm{H}\right), 7.52\left(\mathrm{dt}, J_{1}=7.8 \mathrm{~Hz}, J_{2}=1.1 \mathrm{~Hz}, 1 \mathrm{H}\right), 7.38$ $\left(\mathrm{dd}, J_{1}=7.9 \mathrm{~Hz}, J_{2}=7.9 \mathrm{~Hz}, 1 \mathrm{H}\right), 6.52\left(\mathrm{dd}, J_{1}=14.3 \mathrm{~Hz}, J_{2}=\right.$ $6.8 \mathrm{~Hz}, 1 \mathrm{H}), 4.54-4.58(\mathrm{~m}, 2 \mathrm{H}), 4.24\left(\mathrm{dd}, J_{1}=14.3 \mathrm{~Hz}, J_{2}=2.3\right.$ $\mathrm{Hz}, 1 \mathrm{H}), 4.08\left(\mathrm{dd}, J_{1}=6.8 \mathrm{~Hz}, J_{2}=2.3 \mathrm{~Hz}, 1 \mathrm{H}\right), 4.00-4.04(\mathrm{~m}$, $2 \mathrm{H}) ;{ }^{13} \mathrm{C}$ NMR $\left(300 \mathrm{MHz}, \mathrm{CDCl}_{3}\right) \delta 165.3,151.4,134.5,133.1$, 131.6, 129.7, 127.9, 87.2, 65.7, 63.5; FT-IR (film) 2925, 1722 $(\mathrm{C}=\mathrm{O}), 1621,1427,1257,819,748 \mathrm{~cm}^{-1}$; Ms $m / z(\%) 226\left(\mathrm{M}^{+}\right.$, 1), 185 (33), 183 (94), 141 (36), 139 (100), 111 (58).

3-Chloro-2'-methylbenzophenone (5e): ${ }^{1} \mathrm{H}$ NMR (300 MHz, $\left.\mathrm{CDCl}_{3}\right) \delta 7.78(\mathrm{~d}, J=1.8 \mathrm{~Hz}, 1 \mathrm{H}), 7.66(\mathrm{~d}, J=7.8 \mathrm{~Hz}, 1 \mathrm{H})$, $7.52(\mathrm{~d}, J=7.5 \mathrm{~Hz}, 1 \mathrm{H}), 7.38-7.42(\mathrm{~m}, 2 \mathrm{H}), 7.25-7.31(\mathrm{~m}, 3 \mathrm{H})$, $2.34(\mathrm{~s}, 3 \mathrm{H}) ;{ }^{13} \mathrm{C}$ NMR $\left(300 \mathrm{MHz}, \mathrm{CDCl}_{3}\right) \delta 197.1,139.4,137.8$, 137.0, 134.8, 133.0, 131.2, 130.7, 129.9, 129.8, 128.6, 128.3, 125.5, 20.0; FT-IR (film) 3020, $1666(\mathrm{C}=\mathrm{O}), 1570,1422,1260$, $738,675 \mathrm{~cm}^{-1}$; Ms $m / z(\%) 232\left(\mathrm{M}^{+}+2,16\right), 230\left(\mathrm{M}^{+}, 48\right), 195$ (100), 119 (73), 91 (80).

4-Methoxyacetophenone (5f): ${ }^{1} \mathrm{H} \mathrm{NMR}\left(300 \mathrm{MHz}, \mathrm{CDCl}_{3}\right)$ $\delta 7.94(\mathrm{~d}, J=6.9 \mathrm{~Hz}, 2 \mathrm{H}), 6.93(\mathrm{~d}, J=6.9 \mathrm{~Hz}, 2 \mathrm{H}), 3.87(\mathrm{~s}, 3 \mathrm{H})$, $2.56(\mathrm{~s}, 3 \mathrm{H}) ;{ }^{13} \mathrm{C} \mathrm{NMR}\left(300 \mathrm{MHz}, \mathrm{CDCl}_{3}\right) \delta 196.8,163.5,130.6$, 130.3, 113.7, 55.5, 26.4; FT-IR (film) 3010, 2965, $1673(\mathrm{C}=\mathrm{O})$, $1602,1359,1258,833 \mathrm{~cm}^{-1}$; Ms $m / z(\%) 150\left(\mathrm{M}^{+}, 56\right), 135(100)$, 107 (21), 92 (23), 77 (39).

4-Chloro-4'-methoxybenzophenone (5g): $\mathrm{mp} 126 \sim 127^{\circ} \mathrm{C}$; ${ }^{1} \mathrm{H}$ NMR $\left(300 \mathrm{MHz}, \mathrm{CDCl}_{3}\right) \delta 7.80(\mathrm{~d}, J=6.9 \mathrm{~Hz}, 2 \mathrm{H}), 7.71$ $(\mathrm{d}, J=6.7 \mathrm{~Hz}, 2 \mathrm{H}), 7.45(\mathrm{~d}, J=6.7 \mathrm{~Hz}, 2 \mathrm{H}), 6.97(\mathrm{~d}, J=6.9 \mathrm{~Hz}$, 2H), 3.89 (s, 3H); ${ }^{13} \mathrm{C} \mathrm{NMR}\left(300 \mathrm{MHz}, \mathrm{CDCl}_{3}\right) \delta 194.3,163.4$, $138.3,136.5,132.5,131.2,129.8,128.5,113.7,55.5$; FT-IR $(\mathrm{KBr}) 3025,2924,1640(\mathrm{C}=\mathrm{O}), 1601,1493,1452,1371,1029$, $853,759 \mathrm{~cm}^{-1}$; Ms $m / z(\%) 248\left(\mathrm{M}^{+}+2,16\right), 246\left(\mathrm{M}^{+}, 47\right), 141$ 
(4), 139 (14), 135 (100), 111 (11).

4-Chlorophenyl (2'-furyl) methanone (5h): $\mathrm{mp} 58 \sim 59^{\circ} \mathrm{C}$; ${ }^{1} \mathrm{H} \mathrm{NMR}\left(300 \mathrm{MHz}, \mathrm{CDCl}_{3}\right) \delta 7.95(\mathrm{~d}, J=8.4 \mathrm{~Hz}, 2 \mathrm{H}), 7.71(\mathrm{~s}$, $1 \mathrm{H}), 7.47(\mathrm{~d}, J=8.4 \mathrm{~Hz}, 2 \mathrm{H}), 7.26(\mathrm{~d}, J=3.7 \mathrm{~Hz}, 1 \mathrm{H}), 6.61$ (dd, $\left.J_{1}=3.5 \mathrm{~Hz}, J_{2}=1.6 \mathrm{~Hz}, 1 \mathrm{H}\right) ;{ }^{13} \mathrm{C}$ NMR $\left(300 \mathrm{MHz} \mathrm{CDCl}_{3}\right) \delta$ 181.1, 152.2, 147.2, 139.1, 135.4, 130.8, 128.8, 120.6, 112.4; FT-IR (KBr) 3128, $1651(\mathrm{C}=\mathrm{O}), 1586,1461,1308,1089,961$, $837,754 \mathrm{~cm}^{-1}$; Ms $m / z(\%) 208\left(\mathrm{M}^{+}+2,11\right), 206\left(\mathrm{M}^{+}, 32\right), 141$ (13), 139 (39), 111 (18), 95 (100).

Cyclohexyl (2-thienyl) methanone (5i): ${ }^{1} \mathrm{H}$ NMR $(300 \mathrm{MHz}$, $\left.\mathrm{CDCl}_{3}\right) \delta 7.72\left(\mathrm{dd}, J_{1}=3.8 \mathrm{~Hz}, J_{2}=1.1 \mathrm{~Hz}, 1 \mathrm{H}\right), 7.61\left(\mathrm{dd}, J_{1}=\right.$ $\left.5.0 \mathrm{~Hz}, J_{2}=1.1 \mathrm{~Hz}, 1 \mathrm{H}\right), 7.13\left(\mathrm{dd}, J_{1}=5.0 \mathrm{~Hz}, J_{2}=3.8 \mathrm{~Hz}, 1 \mathrm{H}\right)$, $3.05-3.15(\mathrm{~m}, 1 \mathrm{H}), 1.83-1.94(\mathrm{~m}, 4 \mathrm{H}), 1.72-1.76(\mathrm{~m}, 1 \mathrm{H}), 1.48-$ $1.61(\mathrm{~m}, 2 \mathrm{H}), 1.20-1.45(\mathrm{~m}, 3 \mathrm{H}) ;{ }^{13} \mathrm{CNMR}\left(300 \mathrm{MHz}, \mathrm{CDCl}_{3}\right)$ $\delta 196.8,143.9,133.4,131.4,128.0,47.5,29.6,25.8$ (overlapped); FT-IR (film) 2930, 2853, $1651(\mathrm{C}=\mathrm{O}), 1519,1415$, $1252,719 \mathrm{~cm}^{-1}$; Ms m/z (\%) $196\left(\mathrm{M}^{+}+2,3\right), 194\left(\mathrm{M}^{+}, 74\right), 139$ (34), 126 (40), 113 (13), 111 (100).

4-Methoxyphenyl (2'-thienyl) methanone (5j): $\mathrm{mp} 73 \sim 74{ }^{\circ} \mathrm{C}$; ${ }^{1} \mathrm{H}$ NMR $\left(300 \mathrm{MHz}, \mathrm{CDCl}_{3}\right) \delta 7.90(\mathrm{~d}, J=8.9 \mathrm{~Hz}, 2 \mathrm{H}), 7.68$ $\left(\mathrm{dd}, J_{1}=5.0 \mathrm{~Hz}, J_{2}=1.1 \mathrm{~Hz}, 1 \mathrm{H}\right), 7.64\left(\mathrm{dd}, J_{1}=3.8 \mathrm{~Hz}, J_{2}=1.1\right.$ $\mathrm{Hz}, 1 \mathrm{H}), 7.15\left(\mathrm{dd}, J_{1}=4.9 \mathrm{~Hz}, J_{2}=3.8 \mathrm{~Hz}, 1 \mathrm{H}\right), 6.98(\mathrm{~d}, J=8.9$ $\mathrm{Hz}, 2 \mathrm{H}), 3.89(\mathrm{~s}, 3 \mathrm{H}) ;{ }^{13} \mathrm{C} \mathrm{NMR}\left(300 \mathrm{MHz}, \mathrm{CDCl}_{3}\right) \delta 186.9$, 163.1, 143.8, 134.0, 133.4, 131.6, 130.7, 127.8, 113.7, 55.5; FT-IR (KBr) 3110, 3009, 2969, $1630(\mathrm{C}=\mathrm{O}), 1505,1417,1253$, $1023,835,740 \mathrm{~cm}^{-1}$; Ms $m / z(\%) 220\left(\mathrm{M}^{+}+2,3\right), 218\left(\mathrm{M}^{+}, 80\right)$, 135 (100), 111 (37), 92 (13), 77 (15).

Pheny (3-pyridyl) methanone (5k): ${ }^{1} \mathrm{H}$ NMR $(300 \mathrm{MHz}, \mathrm{CD}$ $\left.\mathrm{Cl}_{3}\right) \delta 9.00(\mathrm{~d}, J=1.5 \mathrm{~Hz}, 1 \mathrm{H}), 8.81\left(\mathrm{dd}, J_{1}=4.8 \mathrm{~Hz}, J_{2}=1.7\right.$ $\mathrm{Hz}, 1 \mathrm{H}), 8.12\left(\mathrm{ddd}, J_{1}=7.9 \mathrm{~Hz}, J_{2}=2.0 \mathrm{~Hz}, J_{3}=2.0 \mathrm{~Hz}, 1 \mathrm{H}\right)$, 7.80-7.84 (m, 2H), 7.61-7.64 (m, 1H), 7.43-7.54 (m, 3H); ${ }^{13} \mathrm{C}$ NMR $\left(300 \mathrm{MHz}, \mathrm{CDCl}_{3}\right) \delta 194.9,152.8,150.9,137.2,136.7$, 133.2, 130.0, 128.6, 128.1, 123.4; FT-IR (film) 3058, 1662 $(\mathrm{C}=\mathrm{O}), 1585,1416,1284,923,713 \mathrm{~cm}^{-1}$; Ms $m / z(\%) 183\left(\mathrm{M}^{+}\right.$, 100), 182 (53), 106 (22), 105 (91), 77 (59).

4-Methylphenyl (3'-pyridyl) methanone (5l): $\mathrm{mp} 76 \sim 77^{\circ} \mathrm{C}$; ${ }^{1} \mathrm{H} \mathrm{NMR}\left(300 \mathrm{MHz}, \mathrm{CDCl}_{3}\right) \delta 8.97-8.99(\mathrm{~m}, 1 \mathrm{H}), 8.80\left(\mathrm{dd}, J_{1}=\right.$ $\left.4.8 \mathrm{~Hz}, J_{2}=1.7 \mathrm{~Hz}, 1 \mathrm{H}\right), 8.10\left(\mathrm{ddd}, J_{1}=7.9 \mathrm{~Hz}, J_{2}=2.0 \mathrm{~Hz}, J_{3}=\right.$ $2.0 \mathrm{~Hz}, 1 \mathrm{H}), 7.74(\mathrm{~d}, J=8.0 \mathrm{~Hz}, 2 \mathrm{H}), 7.44\left(\mathrm{ddd}, J_{1}=7.9 \mathrm{~Hz}\right.$, $\left.J_{2}=4.9 \mathrm{~Hz}, J_{3}=0.8 \mathrm{~Hz}, 1 \mathrm{H}\right), 7.32(\mathrm{~d}, J=8.0 \mathrm{~Hz}, 2 \mathrm{H}), 2.46(\mathrm{~s}$, $3 \mathrm{H}) ;{ }^{13} \mathrm{C}$ NMR $\left(300 \mathrm{MHz}, \mathrm{CDCl}_{3}\right) \delta 194.5,152.6,150.8,144.2$, 137.1, 134.1, 133.5, 130.3, 129.3, 123.3, 21.7; FT-IR (KBr) $3030,1651(\mathrm{C}=\mathrm{O}), 1606,1420,1288,1024,825,741 \mathrm{~cm}^{-1} ; \mathrm{Ms}$ $m / z(\%) 197$ (M+, 98), 182 (52), 119 (100), 106 (18), 91 (59).

2,2-Dimethyl-3-undecanone (5m): ${ }^{1} \mathrm{H}$ NMR $(300 \mathrm{MHz}$, $\left.\mathrm{CDCl}_{3}\right) \delta 2.47(\mathrm{t}, J=7.3 \mathrm{~Hz}, 2 \mathrm{H}), 1.52-1.59(\mathrm{~m}, 2 \mathrm{H}), 1.25-1.32$ $(\mathrm{m}, 10 \mathrm{H}), 1.13(\mathrm{~s}, 9 \mathrm{H}), 0.88(\mathrm{t}, J=6.7 \mathrm{~Hz}, 3 \mathrm{H}) ;{ }^{13} \mathrm{C}$ NMR $(300$ $\left.\mathrm{MHz}_{2} \mathrm{CDCl}_{3}\right) \delta 216.1,44.1,36.4,31.9,29.5,29.4,29.2,26.4$,
24.0, 22.7, 14.1; FT-IR (film) 2926, 2852, 1708 (C=O), 1452, $1365,1070 \mathrm{~cm}^{-1}$; Ms $m / z(\%) 198\left(\mathrm{M}^{+}, 4\right), 142$ (10), 141 (100), 85 (10), 71 (41).

2',4',6'-Trimethyl-2,2-dimethylpropiophenone (5n): ${ }^{1} \mathrm{H}$ NMR $\left(300 \mathrm{MHz} \mathrm{CDCl}_{3}\right) \delta 6.82(\mathrm{~s}, 2 \mathrm{H}), 2.26(\mathrm{~s}, 3 \mathrm{H}), 2.17(\mathrm{~s}, 6 \mathrm{H})$, $1.23(\mathrm{~s}, 9 \mathrm{H}) ;{ }^{13} \mathrm{C}$ NMR $\left(300 \mathrm{MHz}, \mathrm{CDCl}_{3}\right) \delta 219.5,139.6,137.3$, 132.0, 128.4, 44.8, 28.1, 20.9, 20.3; FT-IR (film) 2968, 2871, $1688(\mathrm{C}=\mathrm{O}), 1611,1477,1379,1365,1147,930,851 \mathrm{~cm}^{-1}$; Ms m/z (\%) $204\left(\mathrm{M}^{+}, 1\right), 148$ (30), 147 (100), 119 (56), 91 (23).

Acknowledgments. This research was supported by the Duksung Women's University Research Grants 3000001003 (2009).

\section{References}

1. (a) O'neill, B. T. In Comprehensive Organic Synthesis; Trost, B. M., Ed.; Pergamon Press: Oxford, U. K., 1991; Vol. 1, pp 397. (b) Aldabbagh, F. In Comprehensive Organic Functional Group Transformations II; Katritzky, A. R., Ed.; Elsevier Ltd.: Oxford, U. K., 2005; Vol. 3, pp 267.

2. Sato, F.; Inoue, M.; Oguro, K.; Sato, M. Tetrahedron Lett. 1979, 20, 4303.

3. Maeda, H.; Okamoto, J.; Ohmori, H. Tetrahedron Lett. 1996, 37, 5381.

4. Alonso, D. A.; Najera, C.; Pacheco, M. C. J. Org. Chem. 2004, $69,1615$.

5. Lerebours, R.; Camacho-Soto, A.; Wolf, C. J. Org. Chem. 2005, 70,8601 .

6. Wang, B.; Bonin, M.; Micouin, L. J. Org. Chem. 2005, 70, 6126.

7. Tokuyama, H.; Yokoshima, S.; Yamashita, T.; Fukuyama, T. Tetrahedron Lett. 1998, 39, 3189.

8. Yu, Y.; Liebeskind, L. S. J. Org. Chem. 2004, 69, 3554.

9. Wattanasin, S.; Kathawala, F. G. Tetrahedron Lett. 1984, $25,811$.

10. (a) Sengupta, S.; Mondal, S.; Das, D. Tetrahedron Lett. 1999, 40, 4107. (b) Jackson, M. M.; Leverett, C.; Toczko, J. F.; Roberts, J. C. J. Org. Chem. 2002, 67, 5032. (c) Badioli, M.; Ballini, R.; Bartolacci, M.; Bosica, G.; Torregiani, E.; Marcantoni, E. J. Org. Chem. 2002, 67, 8938 .

11. (a) Nahm, S.; Weinreb, S. M. Tetrahedron Lett. 1981, 22, 3815. (b) Sibi, M. P. Org. Prep. Proced. Int. 1993, 25, 15. (c) Liu, J.; Ikemoto, N.; Petrillo, D.; Armstrong, J. D. Tetrahedron Lett. 2002, 43, 8223 .

12. Qu, B.; Collum, D. B. J. Org. Chem. 2006, 71, 7117.

13. (a) Araki, M.; Sakata, S.; Takei, H.; Mukaiyama, T. Bull. Chem. Soc. Jpn. 1974, 47, 1777. (b) Nicolaou, K. C.; Claremon, D. A.; Papahatjis, D. P. Tetrahedron Lett. 1981, 22, 4647. (c) Vazquez, J.; Albericio, F. Tetrahedron Lett. 2002, 43, 7499.

14. (a) Meyers, A. I.; Comins, D. L. Tetrahedron Lett. 1978, 19, 5179. (b) Comins, D. L. Synlett 1992, 615.

15. Abe, K.; Sato, T.; Nakamura, N.; Sakan, T. Chem. Lett. 1977, 645.

16. (a) Heras, M. A.; Molina, A.; Vaquero, J. J.; Navio, J. L. G.; Alvarez-Builla, J. J. Org. Chem. 1993, 58, 5862. (b) Heras, M. A.; Vaquero, J. J.; Navio, G.; Alvarez-Builla, J. Tetrahedron 1996, $52,14297$.

17. Lee, J. I. Bull. Korean Chem. Soc. 2007, $28,863$. 